\title{
The effect of cognitive impairment on self-generation in Hispanics with TBI
}

\author{
Carlos José De los Reyes Aragón ${ }^{\mathrm{a}, *}$, Juan Carlos Arango-Lasprilla ${ }^{\mathrm{b}}$, María Perea Bartoloméc, \\ Valentina Ladera Fernández ${ }^{\mathrm{c}}$ and Denise $\mathrm{Krch}^{\mathrm{d}}$ \\ ${ }^{a}$ Departmen of Psychology Universidad del Norte, Barranquilla Colombia, Barranquilla, Colombia \\ ${ }^{\mathrm{b}}$ Department of Physical Medicine and Rehabilitation Virginia Commonwealth University, Richmond, VA, USA \\ ${ }^{\mathrm{c}}$ Department of Basic Psychology, Psychobiology and Behavioral Sciences' Methodology, Universidad de \\ Salamanca, Salamanca, Spain \\ ${ }^{\mathrm{d}}$ Kessler Foundation Research Center, West Orange, NJ, USA
}

\begin{abstract}
The objective of this study was to determine the moderating effect of cognitive impairment (CI) on the usefulness of the generation effect to improve learning and memory in Hispanics with traumatic brain injury (TBI). Sixty-one Hispanic individuals with TBI (29 without CI, 22 with mild to moderate CI, and 10 with severe CI) and 44 healthy controls (HC) were required to remember the last word in each of 32 sentences. Target words were presented in a self-generated and provided condition. Recall and recognition were examined immediately, after 30 minutes, and at one week. Individuals remembered and recalled significantly more words in the generated condition than the provided condition, regardless of group or time. The self-generation technique equally benefitted all participants regardless of TBI status or degree of CI. Future cognitive rehabilitation programs designed to improve short-term recall and recognition in Hispanic individuals with TBI should include the self-generation technique. Further research into the longer-term effects of the generation effects is warranted.
\end{abstract}

Keywords: Traumatic brain injury, self-generation technique, cognitive rehabilitation

\section{Introduction}

The generation effect (GE) is a phenomenon based on the idea that self-generated information is more easily remembered than that which is externally provided $[3,24,42]$, suggesting that active participation on the part of the learner leads to greater retention than passive participation.

Many variations to the GE paradigm exist, however the basic model consists of the presentation of list of associated word pairs (e.g., synonyms, antonyms, or mathematical equations). Half of the pairs are provided complete by the examiner, who instructs the subject to simply read the pair [9], whereas the remaining pairs are presented without the second word in the pair, which

\footnotetext{
*Corresponding author: Carlos José De los Reyes Aragón, Departamento de Psicología, Universidad del Norte. Km 5 Vía Puerto Colombia. Barranquilla (Colombia), Barranquilla, Colombia. Tel.: +57 5 3509291; E-mail: cdelosreyes@uninorte.edu.co.
}

the subject is required to generate. Studies [21,27,42] show that individuals remember more of the words that they have generated themselves than those that have been provided to them. Other variations include the presentation of sentences for which the last word is provided versus generated [40], presenting multiplication [39], or addition tasks [31], or presenting anagrams with intact or scrambled solutions [18].

In recent years, numerous investigations have demonstrated that the GE is useful for learning different types of information, such as words $[7,8,13,43,45]$, texts [16], numbers [15], pictures [26], contextual features [29], acronyms [19] and pseudo-words [25]. Research has demonstrated the utility of the GE in healthy adults $[8,11,20,38,42,45]$, as well as in individuals with various neurological conditions, such as dementia [5, $34,44]$ and multiple sclerosis [13,36].

There are only a handful of studies which have investigated how the GE may improve new learning and memory in individuals with traumatic brain in- 
jury (TBI). A study by Lengenfelder, Chiaravalloti, and Deluca [27], examined the usefulness of the GE in 18 subjects with moderate to severe TBI and $18 \mathrm{HCs}$. Participants were presented with 32 sentences, half of which were simply read, and half of which required the last word to be generated. In half of the sentences, the last word was omitted, leaving a blank space for subjects to fill in with a word they self-generated. In the other half of the sentences, the last word appeared underlined. Subsequently, subjects were asked to recall and recognize the provided and generated words immediately, after 30 minutes and one week delay. The results showed that $\mathrm{HCs}$ showed greater benefit from the self-generation technique, however both participants with TBI as well as the HCs had better recall of self-generated words than provided words. The benefit of the GE diminished over time for both groups, with similar rates of forgetting at 30-minute and one-week delayed testing.

In another study, Goverover, Chiaravallotti, DeLuca and Johnston [21] examined the usefulness of the GE as a method to improve the learning and memory of everyday functional tasks in patients with TBI. This study included 10 subjects with TBI and $15 \mathrm{HCs}$, all of whom were instructed in 2 cooking tasks and 2 financial management tasks. In the course of their instruction, one of the cooking tasks and one of the financial management tasks were taught through the use of step-by-step instructions, which were simply read (provided condition). In the other cooking and finance tasks, subjects were provided instructions with key words missing and were asked to fill in the missing information (generated condition). The subjects' ability to recall the learned tasks as well as remembering all the steps involved was evaluated immediately, 30 minutes later, and again after one week. The results showed that tasks which were taught using the GE were substantially better remembered in both groups with no significant group effects. Benefits were maintained for both groups one week following training.

Schefft et al. [41] evaluated the effectiveness of the GE by having participants learn a list of associated word pairs across two experiments. In the first experiment, free recall and recognition were assessed in 20 individuals with TBI. In the second experiment, free and cued recall were assessed in a distinct sample of 20 individuals with TBI. The results demonstrated that the GE did not improve free recall of information in either experiment. However, the GE did improve recognition memory and cued recall, with varying degrees of benefit from cueing depending on the type of cue utilized.
Similarly, O'Brien and colleagues [36] investigated the utility of the GE in improving recall in 18 individuals with TBI and 31 with multiple sclerosis (MS) by using the same paradigm as Lengenfelder et al. [27]. However, they additionally considered the influence of cognitive impairment (CI) on the participants' ability to benefit from the GE. In this study, the level of CI was determined using a comprehensive neuropsychological battery, and participants were classified as impaired or unimpaired in each of three domains: working memory, episodic memory, and executive functions. Results revealed that, although the patients with impairments in one or less cognitive domains benefitted from the generation technique, the benefit to patients with multiple impairments was reduced. The results also showed that the patients with TBI as well as those with MS benefited from the self-generation technique at immediate and 30-minute delayed, but not after one week. Individuals without working memory impairments benefited more from the self-generation method relative to those with such impairments, whereas no such benefit was seen for persons who were free of episodic memory and executive functions impairments as compared to those with such impairments.

Recent studies have demonstrated preliminary evidence of the utility of the GE in TBI, however some of these findings are mixed. Whereas Lengenfelder et al. [27] found that individuals with TBI benefitted from the GE on recall and recognition at immediately and 30 delay. Shefft et al. [41] found that individuals with TBI benefitted from the GE on cued recall and recognition tasks, but not on free recall. Although the individuals that participated in both of these studies sustained primarily moderate to severe TBI, their CI is unknown and as such, conclusions cannot be drawn regarding their degree of CI and their ability to benefit from the GE.

Research in neurological populations (e.g. [5,34,44]) suggests that the degree of benefit derived from GE varies by the severity of cognitive deficits. A recent study in TBI, conducted by O'Brien and colleagues, revealed similar findings [36]. In O'Brien's study, severity of CI was determined by the number of impaired cognitive domains (i.e., complex working memory, episodic memory, and executive functioning) as defined by a $z$-score of $\leqslant-1.00$. This approach may have produced a heterogeneous CI sample in two ways. Individuals with impairments in diverse cognitive domains were pooled into a single group with individuals whose type of cognitive impairments may have been different. An additional source of heterogeneity arises from the liberal cutoff for impairment, which leads to group- 
Table 1

Sociodemographic and injury characteristics

\begin{tabular}{lccccc}
\hline Variable & HC & TBI Wo CI & TBI M/M CI & TBI S CI & $p$ \\
& $n=44$ & $n=29$ & $n=22$ & $n=10$ & \\
\hline Age (SD) & 32.70 & 32.14 & 34.50 & 40.50 & NS \\
& $(12.37)$ & $(9.99)$ & $(11.04)$ & $(13.68)$ & \\
Years of education (SD) & 13.16 & 11.90 & 10.77 & 9.80 & $<0.05$ \\
& $(3.32)$ & $(3.99)$ & $(2.99)$ & $(4.02)$ & \\
Gender (\%) & & & & & NS \\
Male & 63.6 & 65.5 & 68.2 & 70.0 & \\
Female & 36.4 & 34.5 & 31.8 & 30.0 & \\
Handedness (\%) & & & & & NS \\
Right-handed & 95.5 & 96.6 & 90.9 & 100.0 & \\
Left-handed & 4.5 & 3.4 & 9.1 & 0.0 & \\
GCS (SD) & - & 10.50 & 9.67 & 9.17 & $\mathrm{NS}$ \\
& & $(3.10)$ & $(2.47)$ & $(3.43)$ & \\
Time since injury in & - & 22.62 & 21.14 & 40.60 & $\mathrm{NS}$ \\
months (SD) & & $(30.53)$ & $(27.84)$ & $(91.35)$ & \\
\hline
\end{tabular}

HC: Healthy controls; TBI Wo CI: Traumatic brain injury participants without cognitive impairment; TBI M/M CI: Traumatic brain injury participants with mild/moderate cognitive impairment: TBI S CI: Traumatic brain injury participants with severe cognitive impairment; SD: Standard deviation; NS: Non significant; GCS: Glasgow Coma Scale.

ing together individuals who may have varied widely in severity of cognitive impairment. These sources of heterogeneity may complicate interpretation of the results. Similar to O'Brien's research. The goal of the current study was to evaluate the influence of the degree of cognitive impairment on the ability to benefit from the self-generation technique to improve the learning and memory of Hispanics with traumatic brain injuries. However, in expanding upon previous research, cognitive impairment in this study will be evaluated by performance on a single measure of global cognitive functioning, with severity determined by performance on this measure.

\section{Methods}

\subsection{Participants}

The study sample included 61 individuals with TBI, who were seen as outpatients at the Hospital of the Universidad del Valle (Cali) or the Cervantes Clinic (Barranquilla) in Colombia. Diagnosis of TBI was confirmed by hospital medical records; time since injury was at least six months. The study also included 44 HC participants, who were recruited from the community through flyers and word-of-mouth. Attempts were made to recruit HCs that matched the TBI group as closely as possible on socio-demographic characteristics. Participants were included in the study if they were between the ages of 18 and 65 years old and had greater than 5 years of education. They were excluded if they had a family history of psychiatric or psychological disorders, drug or alcohol abuse, or learning disabilities or aphasia at the time of the study.

Demographics and injury characteristics of the two groups are presented in Table 1. The groups were not significantly different in terms of age, gender, or handedness, however the HC group was significantly more educated $(p<0.05)$ than the TBI group.

\subsection{Procedure}

After the Ethics Committee of the Universidad del Norte in Barranquilla evaluated and approved the study, the participants who met the inclusion criteria were contacted via telephone and invited to take part in the study. Interested individuals were scheduled for a neuropsychological evaluation, which included administration of the self-generation procedure. Written informed consent was obtained from participants after the study was explained and all questions had been answered. The neuropsychological evaluation lasted approximately 45 minutes, after which the self-generation procedure was conducted. Recall and recognition for both learning conditions (generated and provided) were evaluated at the time of the appointment, after $30 \mathrm{~min}-$ utes and one week later by telephone.

\subsubsection{Neuropsychological evaluation}

Socio-demographic and medical information were obtained through interview prior to testing. All participants were administered the Spanish Brief Neuropsychological Evaluation (NEUROPSI) [37], a battery that 
has been used in many studies to evaluate global cognitive functioning in individuals with acquired brain injuries (ABI) [4,32]. The NEUROPSI evaluates temporal and spatial orientation, attention, concentration, memory, language, visuospatial cognition, executive functions, literacy (reading and writing), and calculations. Total scores range from 2 to 130, with higher scores indicating better cognitive functioning.

To determine the degree of CI for each TBI participant, NEUROPSI total scores were referenced in age- and education-based normative tables, which provide translation to the classification of degree of cognitive impairment (i.e., intact, mild, moderate, and severe). Mildly and moderately impaired individuals were grouped together in the current study due to the limited number of individuals with mild cognitive impairment. The resulting three subgroups included: TBI Without Cognitive Impairment (TBI Wo CI), TBI with Mild/Moderate Cognitive Impairment (TBI M/M CI), and TBI with Severe Cognitive Impairment (TBI S CI).

\subsubsection{Self-generation procedure}

The self-generation procedure used in this study was a Spanish adaptation [2] of the Multhaup and Balota protocol [34]. The procedure consists of a series of 32 sentences, with half of the sentences from the selfgeneration condition and half from the provided condition. In the self-generation condition, the last word was missing, and subjects were instructed to think of the word they felt was most adequate to complete the sentence. In the provided condition, the sentence had already been completed and the correct word underlined; subjects were asked to read the sentence and remember the last word. The two sentence conditions (i.e., selfgeneration and provided) were alternatively presented to participants in a acounterbalanced manner. Following presentation of all sentences, recall and recognition for both conditions were evaluated immediately, and at 30-minute and one-week delays. The generated and provided word lists were matched for age of acquisition and use frequencies based on Spanish language norms, as has been cited elsewhere [2].

\subsection{Statistical analysis}

A $4 \times 2 \times 3$ repeated-measures analyses of covariance (ANCOVA) was performed to compare the four groups (HC, TBI Wo CI, TBI M/M CI, and TBI S CI), across the two testing conditions (self-generated and provided), at three different times (immediate, $30 \mathrm{~min}$ utes, and 1 week), after controlling for education. This model was used to separately analyze performance for recall and recognition. The dependent variable for each analysis was the total number of words correct. Post hoc analyses were conducted to determine where the differences occurred among the groups. Due to the number of post-hoc analyses conducted and to control for the overall Type I error rate, t-test analysis were conducted with significance level of $\alpha=0.01$.

In order to more saliently evaluate the degree of benefit gained from self-generation, a percent benefit score was calculated. Using methods proposed by Bertsch et al. [9] percentage of words generated minus percentage of words provided was calculated for each participant. For example, if a person recalled 12 out of 16 self-generated words $(75 \%)$, and recalled 8 out of 16 provided words $(50 \%)$, the benefit gained through the GE method is $25 \%(75 \%-50 \%)$. Using percent benefit scores, a $4 \times 3$ repeated-measures ANCOVA was performed to compare the four groups (HC, TBI Wo CI, TBI M/M CI, and TBI S CI) at 3 different times (immediate, 30 minutes, and 1 week), after controlling for education. This model was used to separately analyze the percent benefit for recall and recognition.

\section{Results}

\subsection{Free recall}

The results of the repeated-measures ANCOVA revealed a main effect of Group (see Fig. 1), $F_{(3,100)}=$ 18.75, $p<0.001$. Post hoc analyses indicated that the HCs remembered significantly more words than each of the three TBI groups (all $p^{\prime}$ s $<0.001$ ). Simple effects analysis revealed that the TBI group with severe CI recalled significantly fewer words than the TBI group with mild to moderate CI $(p<0.05)$ and the TBI group without CI $(p<0.01)$. There was a Condition $\mathrm{x}$ Time interaction (see Fig. 2), $F_{(2,99)}=$ 5.28, $p<0.01$, such that individuals in the generated condition performed significantly better than those in the provided condition at immediate testing $(t=8.54$, $p<0.001)$; at 30 minutes, performance by individuals in the generated condition remained significantly better than those in the provided condition $(t=6.15$, $p<0.001$ ), however whereas individuals in the generated condition performed similarly from immediate to 30 minute testing ( $t=-0.91, p=\mathrm{NS})$, individuals in the provided condition performed significantly better at 30 minutes compared to immediately $(t=-3.49, p<$ $0.001)$. At 1 week, both groups showed a significant 


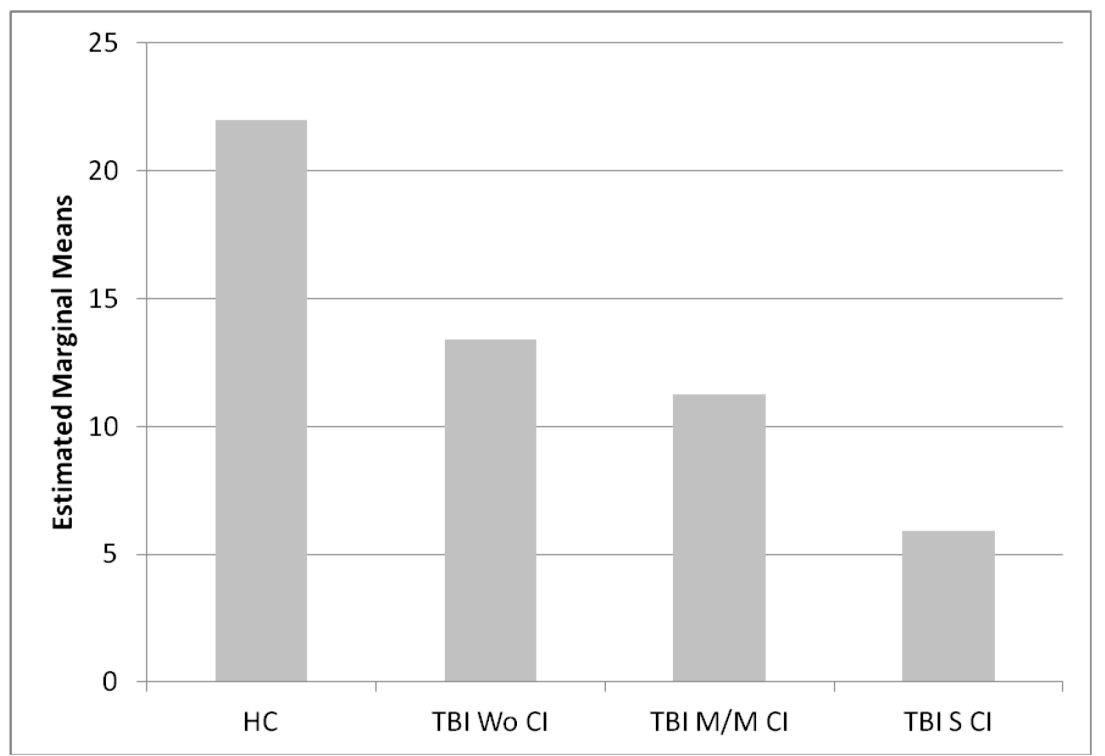

Fig. 1. Number of words correctly recalled. HC: Healthy controls; TBI Wo CI: Traumatic brain injury participants without cognitive impairment; TBI M/M CI: Traumatic brain injury participants with mild/moderate cognitive impairment: TBI S CI: Traumatic brain injury participants with severe cognitive impairment.

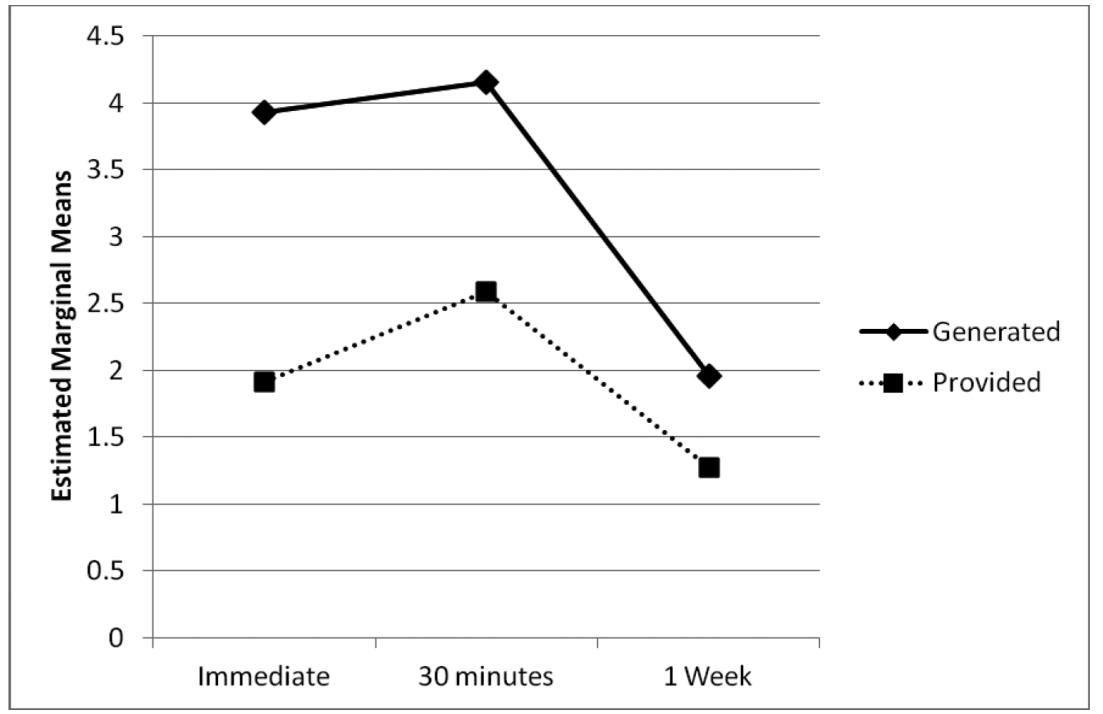

Fig. 2. Number of words correctly recalled across time.

decrease in the number of words recalled relative to both immediate and 30 minutes $\left(p^{\prime} s<0.001\right)$, however performance by individuals in the generated condition remained significantly better than that of individuals in the provided condition $(t=3.88, p<0.001)$. There were no other main effects or interactions observed. That individuals performed significantly better in the generated versus provided condition at all time points supports the presence of a generation effect.

\subsection{Recognition}

The results of the repeated-measures ANCOVA demonstrated a main effect for Group, $F_{(3,100)}=4.80$, $p<0.01$. The TBI group with severe CI recognized significantly fewer words than all other groups $\left(p^{\prime} s<\right.$ 0.01 , while all other groups recognized similar numbers of words regardless of time and condition $\left(p^{\prime} s=\right.$ NS). A main effect was found for Condition, $F_{(1,100)}=$ 


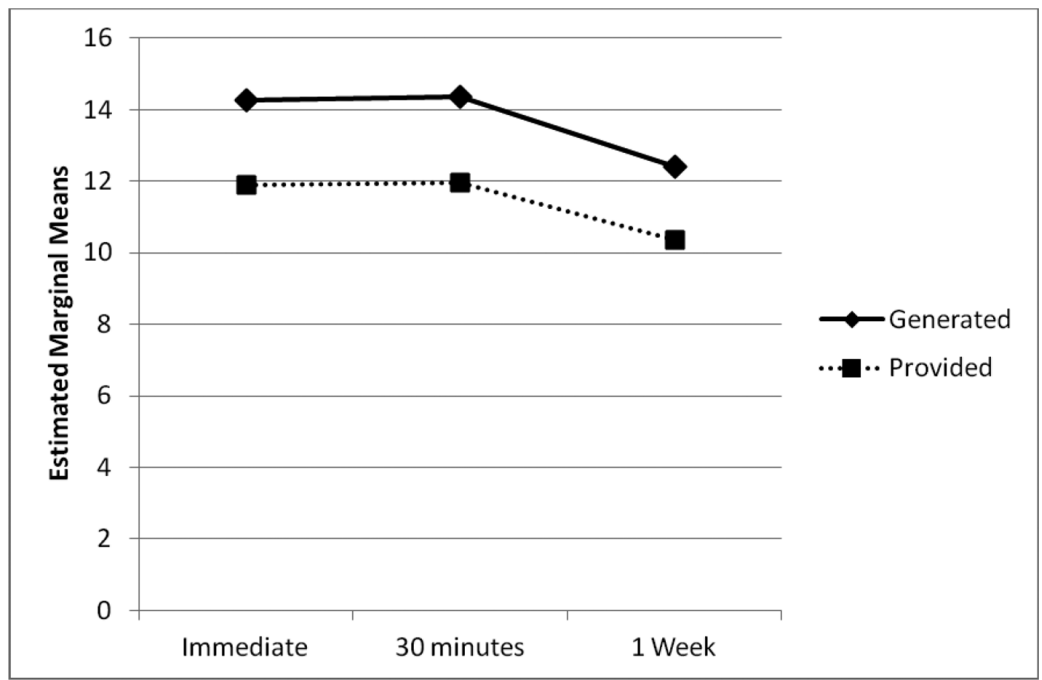

Fig. 3. Number of words correctly recognized across time.

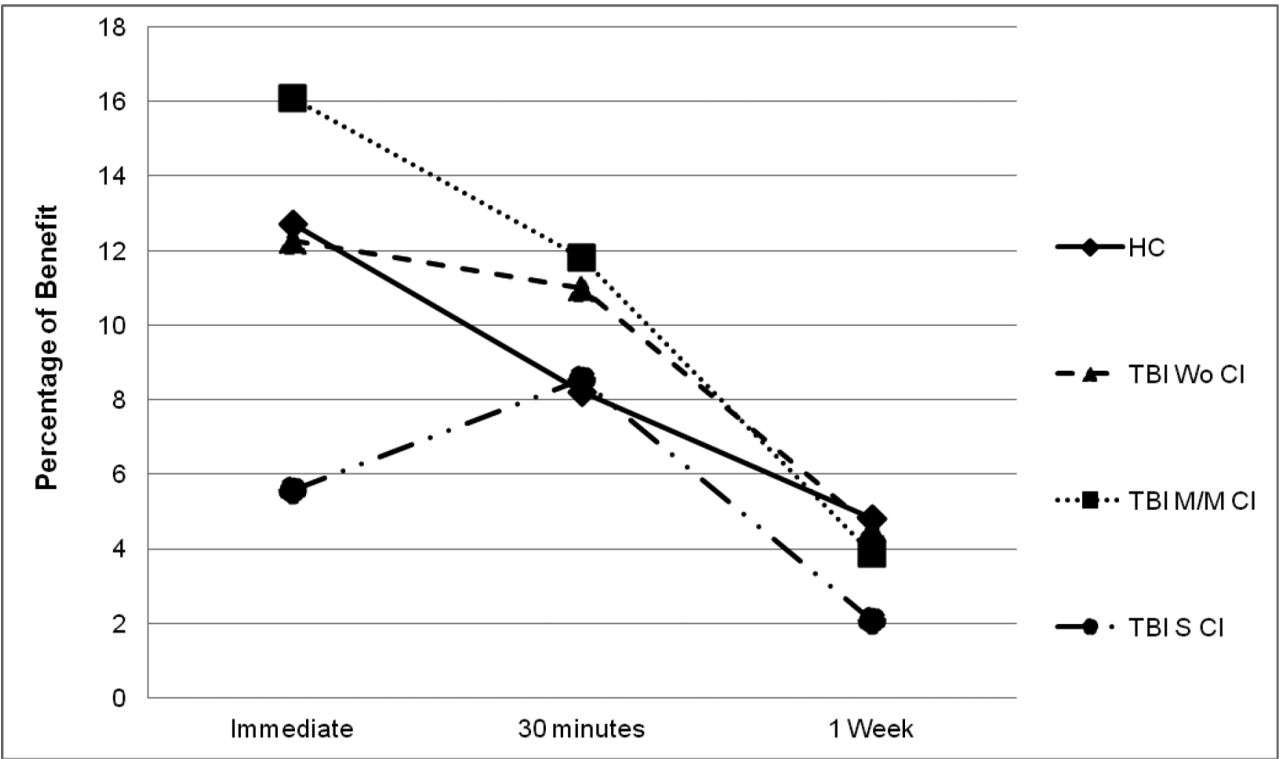

Fig. 4. Percentage benefit of generation effect on recall. $\rightarrow-$ HC: Healthy controls; $-\downarrow$ TBI Wo CI: Traumatic brain injury participants without cognitive impairment; TBI M/M CI: ...... Traumatic brain injury participants with mild/moderate cognitive impairment: TBI S CI: Traumatic $\longrightarrow$. brain injury participants with severe cognitive impairment.

$20.10 ; p<0.001$, such that participants recognized significantly more self-generated than provided words, regardless of group or time. This main effect supports the presence of a generation effect. A main effect for Time was observed, $F_{(2,200)}=7.22, p<0.01$, with the number of words recognized significantly reduced after one week relative to both immediate and 30-minute testing $\left(p^{\prime} s<0.001\right)$. (See Fig. 3). No interactions were observed.

\subsection{Benefits of self-generation for recall memory}

The results of the $4 \times 3$ repeated-measures ANCOVA showed the presence of a main effect for Time, $F_{(2,200)}=5.50, p<0.01$, such that the benefits of the GE method were significantly diminished after oneweek relative to both immediate and 30 -minute testing, $p^{\prime} s<0.01$. (See Fig. 4). There was no main effect observed for Group, suggesting that the self-generation technique equally benefitted all participants, regardless 


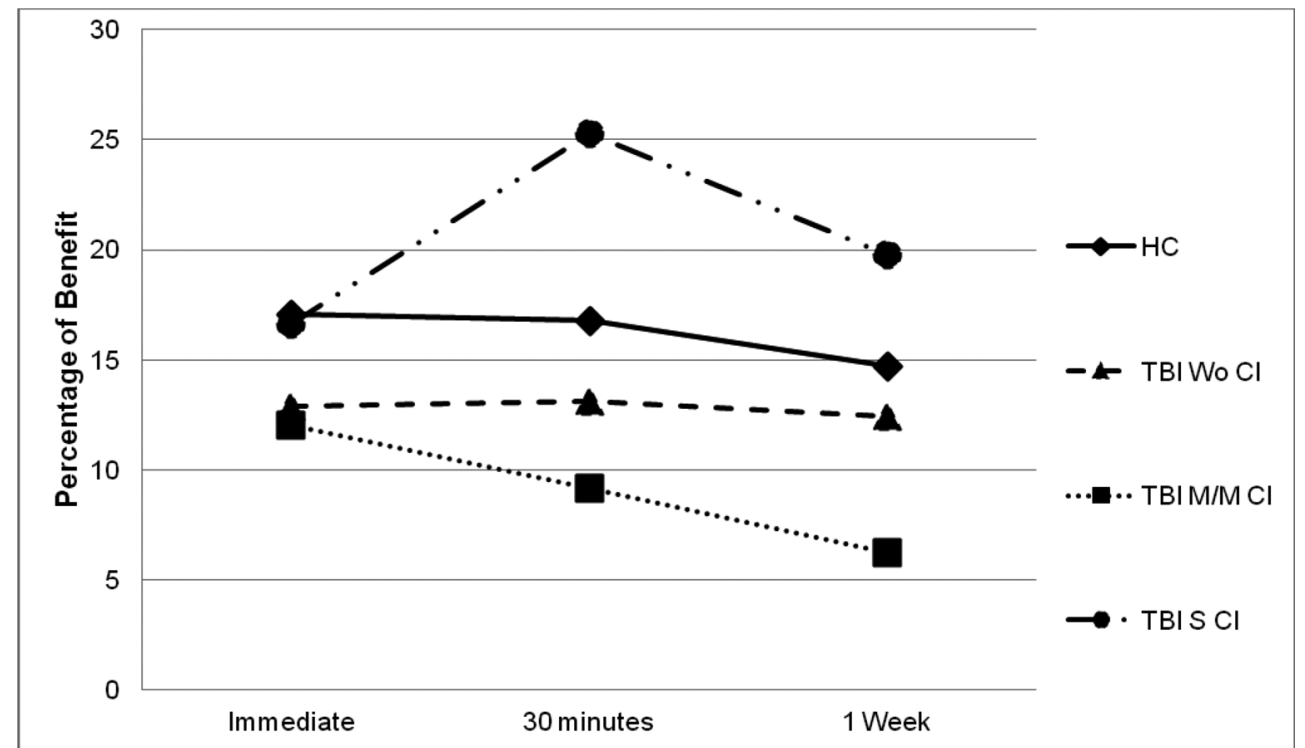

Fig. 5. Percentage benefit of generation effect on recognition. $\neg$ HC: Healthy controls; $-\llcorner$ TBI Wo CI: ...๑.. Traumatic brain injury participants without cognitive impairment; TBI M/M CI: Traumatic $\rightarrow$. brain injury participants with mild/moderate cognitive impairment: TBI S CI: Traumatic brain injury participants with severe cognitive impairment.

of TBI status or the degree of CI. The Group x Time interaction was not significant.

\subsection{Benefits of self-generation for recognition memory}

The results of the $4 \times 3$ repeated-measures ANCOVA did not show any main effects, nor interactions (see Fig. 5), signifying that the benefits of self generation for recognition were similar across all groups and time points.

\section{Discussion}

The objective of this study was to determine the influence of the degree of cognitive impairment (CI) on the benefits gained from the self-generation method to improve learning and memory in Hispanics with traumatic brain injuries. The results suggest that the selfgeneration technique is useful for improving recall and recognition in TBI patients with varying degrees of CI. These results are consistent with other studies investigating the GE's benefit on recall $[27,36]$, recognition [41], and everyday tasks [21] in individuals with TBI. The results additionally add to the existing literature on the GE in other neurological populations $[13,17$, $21,34,36]$, and in healthy subjects $[28,29,35,42]$, which has shown that self-generation is useful in improving memory of new information.

GE was found to significantly benefit performance on free recall and recognition in the current study. Although Lengenfelder and colleagues [27] produced similar findings, Schefft et al. [41] found that the GE benefited recognition and cued recall for information, but not free recall. Schefft et al. concluded that the method of stimuli presentation used in self-generation paradigms influences how well the information is retained. In the current study and in Lengenfelder's work, sentence completion paradigms were used; this is in contrast to Schefft's use of a word pair paradigm. The sentence completion paradigm may provide more contextual features as clues toward self-generation than the associative pairs paradigm. This is consistent with previous research, which shows that the more distinctive features the stimuli possesses, the better the recollection for that information $[10,22,23]$. In fact, it has been demonstrated that the self-generation paradigm used in the current study is the one that shows the most robust results in terms of learning new information [9].

The present study revealed that, although diminished over time, the benefits of self-generation remained significant at 1 week. Previous research has suggested the decreased ability to retain information is due to the lack of personal relevance to participants, who then require repetition or review to retain information. This phenomenon has been documented widely $[12,30,46]$ 
and is supported by Goverover et al.'s [21] GE work in TBI. Specifically, when the GE was applied to everyday tasks, which are significant and relevant to participants, Goverover et al. [21] showed that the benefits of the self-generation technique persisted over a longer period of time. However, the current study revealed a significant benefit at 1 week using a sentence completion paradigm; this is in contrast to other sentence completion studies that revealed the GE benefit lost at 1 week $[27,36]$. The difference between the current study and others sentence completion studies may be attributed to the current study's selection of stimuli items matched for word frequency and age of word acquisition across generated and provided conditions. It is possible that unbalanced stimuli between conditions could lead to biased results. To ensure equivalent difficulty across conditions, future studies should evaluate these characteristics in the stimuli across conditions. Understanding the reasons behind maintenance and loss of gains over time is important if the GE technique is to be a viable cognitive rehabilitation tool.

An important clinical implication of the current study is the fact that, regardless of the degree of CI, TBI patients' learning and memory was improved through the use of the self-generation method. Therefore, the GE may serve as a useful rehabilitation method for a widerange of individuals with TBI. Indeed, results from acquired brain injury studies $[6,7]$ demonstrate that the self-generation method benefits patients with varying severities of CI. In contrast, O'Brien et al. [36] found that MS and TBI subjects with higher degrees of CI benefited less from the GE than those with less or no CI. These contradictory findings may be explained by differences in the way CI was evaluated and defined in each study. Whereas O'Brien and colleagues established CI based on impairment in different cognitive domains (i.e., working memory, episodic memory, and executive functions), the current study established the degree of CI based on global cognitive performance. It should be noted that although O'Brien and colleagues' severe CI group recalled less words, the effect size was large, suggesting that ability to detect benefits in this group may have actually been due to small sample size. One last point of interest is that in O'Brien's study, individuals without specific working memory impairments benefited more from self-generation, indicating that intact working memory may be a requisite for using the $\mathrm{GE}$ as a rehabilitation tool. It will be important for future research to consider not only the degree, but the type of $\mathrm{CI}$ as a factor in understanding the usefulness of the self-generating method.
Because Hispanics in the current sample successfully benefited from a technique that has been proven beneficial in Anglo samples, the GE technique may be readily applied to the cognitive rehabilitation of memory in patients with TBI and other types of $\mathrm{ABI}$ in Latin America. Further, this technique may also be used with Hispanics residing in the U.S. The design of clinical treatment programs which take into consideration mnemonic changes that often arise as a consequence of TBI may lead to a better quality of life for Hispanic patients with TBI or ABI and their families $[1,14,33]$.

The results of the current study should be interpreted in light of several limitations. The recognition task utilizes a two-alternative forced choice format, wherein there is a $50 \%$ probability of obtaining a correct response through guessing. This format likely places less demand on retrieval and in turn, may only be loosely reflective of actual memory functioning. Therefore, future GE studies should consider assessing recognition with a four-alternative forced choice format. Finally, a drawback of the paradigm used in the current study is the utilization of a sentence completion paradigm, which may not have practical application for individuals. The ultimate goal of cognitive rehabilitation is to improve an individuals' ability to function in their own environment and as such, selection of GE paradigms more reflective of real-life activities may result in more robust long-term benefit and greater overall functionality.

\section{Conclusions}

Hispanic patients with TBI benefit from learning and memory of new information through the use of the selfgeneration technique. This benefit was derived regardless of the degree of CI, suggesting that the GE is a viable treatment option for individuals across a wide range of cognitive capacity. Although diminished over time, the GE benefit was not lost at 1 week. Although these findings are promising, the specific characteristics of this paradigm that contributed to maintenance is unclear. Future work should focus on verifying critical factors promote long-term gains. Once these factors have been identified, future studies may evaluate if benefits of GE can be maintained beyond a week. Such studies may find that benefits gained from the GE can be maximized by combining this treatment with other proven techniques, such as visual imagery, errorless learning and pharmacological therapy, among others. 


\section{References}

[1] S. Andren and S. Elmstahl, Family caregivers' subjective experiences of satisfaction in dementia care: aspects of burden, subjective health and sense of coherence, Scand J Caring Sci 19(2) (2005), 157-68.

[2] J. Arango-Lasprilla, N. Chiaravalloti, J. Balzano, G. Voelbel, H. Rogers, F. Cuetos and J. DeLuca, The usefulness of self-generation to improve learning and memory in Hispanics, Journal of Minority Disability Research and Practice 1 (2009), 47-71.

[3] J. C. Arango-Lasprilla, P. Premuda and M. Marquine, Rehabilitación cognitiva en personas con traumatismo craneoencefálico, In: Rehabilitación neuropsicológica, J. C. Arango-Lasprilla, ed. Manual Moderno, Mexico, D.F., 2006, pp. 117-43.

[4] A. Ardila, F. Ostrosky-Solis, M. Rosselli and C. Gómez, AgeRelated Cognitive Decline During Normal Aging: The Complex Effect of Education, Archives of Clinical Neuropsychology 15(6) (2000), 495-513.

[5] A. M. Barrett, G. P. Crucian, R. L. Schwartz and K. M. Heilman, Testing memory for self-generated items in dementia: method makes a difference, Neurology 54(6) (2000), 1258-64.

[6] M. R. Basso, C. Ghormley, N. Lowery, D. Combs and R. A. Bornstein, Self-generated learning in people with multiple sclerosis: an extension of Chiaravalloti and DeLuca (2002), J Clin Exp Neuropsychol 30(1) (2008), 63-9.

[7] M. R. Basso, N. Lowery, C. Ghormley, D. Combs and J. Johnson, Self-generated learning in people with multiple sclerosis, J Int Neuropsychol Soc 12(5) (2006), 640-8.

[8] I. Begg, E. Vinski, L. Frankovich and B. Holgate, Generating makes words memorable, but so does effective reading, Mem Cognit 19(5) (1991), 487-97.

[9] S. Bertsch, B. J. Pesta, R. Wiscott and M. A. McDaniel, The generation effect: a meta-analytic review, Mem Cognit 35(2) (2007), 201-10.

[10] C. Brainerd, V. Reyna and R. Kneer, False recognition reversal: When similarity is distinctive, Journal of Memory and Language 34 (1995), 157-85.

[11] S. E. Clark, The generation effect and the modeling of associations in memory, Mem Cognit 23(4) (1995), 442-55.

[12] F. Craik and E. Tulving, Depth of processing and the retention of words in episodic memory, Journal of Experimental Psychology-General 104 (1975), 268-94.

[13] N. D. Chiaravalloti and J. Deluca, Self-generation as a means of maximizing learning in multiple sclerosis: an application of the generation effect, Arch Phys Med Rehabil 83(8) (2002), 1070-9.

[14] C. De los Reyes-Aragón, Editorial: Prevención e intervención del síndrome de Burnout en daño cerebral, Psicología Desde el Caribe 24 (2009), vii-X.

[15] A. Derwinger, A. Stigsdotter Neely and L. Backman, Design your own memory strategies! Self-generated strategy training versus mnemonic training in old age: an 8-month follow-up, Neuropsychol Rehabil 15(1) (2005), 37-54.

[16] G. Einstein, M. McDaniel, C. Bowers and D. Stevens, Memory for prose: The influence of relational and proposition-specific encoding, Journal of Experimental Psychology: Learning, Memory and Cognition 10 (1984), 133-43.

[17] P. Flory and L. Pring, The effects of data-driven and conceptually driven generation of study items on direct and indirect measures of memory, Q J Exp Psychol A 48(1) (1995), 153-65.

[18] J. Gardiner, A. Dawson and E. Sutton, Specificity and generality of enhanced priming effects for self-generated study items,
American Journal of Psychology 102 (1989), 205-305.

[19] J. Gardiner and J. Hampton, Semantic memory and the generation effect: Some tests of the lexical activation hypothesis, Journal of Experimental Psychology: Learning, Memory and Cognition 11 (1985), 732-41.

[20] J. M. Gardiner and J. M. Rowley, A generation effect with numbers rather than words, Mem Cognit 12(5) (1984), 443-5.

[21] Y. Goverover, N. Chiaravalloti, J. DeLuca and M. Johnston, Self-generation to Improving Learning of Everyday Functional Tasks in Multiple Sclerosis and Traumatic Brain Injury, Journal of de International Neuropsychological Society 11(S1) (2005), 49.

[22] M. L. Howe, When distinctiveness fails, false memories prevail, J Exp Child Psychol 71(2) (1998), 170-7.

[23] R. Hunt, Two contributions of distinctive processing to accurate memories, Journal of Language and Memory 48 (2003), 811-25.

[24] L. Jacoby, On interpreting the effects of repetition: Solving a problem versus remembering a solution, Journal of Verbal Learning \& Verbal Behavior 17 (1978), 649-67.

[25] E. Johns and L. Swanson, The generation effect with nonwords, Journal of Experimental Psychology: Learning, Memory and Cognition 14 (1988), 180-90.

[26] H. Kinjo and J. G. Snodgrass, Does the generation effect occur for pictures? Am J Psychol 113(1) (2000), 95-121.

[27] J. Lengenfelder, N. Chiaravalloti and J. Deluca, Efficacy of the Generation Effect in Improving New Learning in Persons With Traumatic Brain Injury, Rehabilitation Psychology 52(3) (2007), 290-6.

[28] E. J. Marsh, When does generation enhance memory for location? J Exp Psychol Learn Mem Cogn 32(5) (2006), 1216-20.

[29] E. J. Marsh, G. Edelman and G. H. Bower, Demonstrations of a generation effect in context memory, Mem Cognit 29(6) (2001), 798-805.

[30] D. McMurray and J. McIntyre, Depth of processing: Is there a semantic continuum? Australian Journal of Psychology 33(2) (1981), 197-213.

[31] D. S. McNamara and A. F. Healy, A Procedural Explanation of the Generation Effect for Simple and Difficult Multiplication Problems and Answers, Journal of Memory and Language 43(4) (2000), 652-79.

[32] S. Mejia, L. M. Gutierrez, A. R. Villa and F. Ostrosky-Solis, Cognition, Functional Status, Education, and the Diagnosis of Dementia and Mild Cognitive Impairment in SpanishSpeaking Elderly, Applied Neuropsychology 11(4) (2004), 194-201.

[33] A. Moreno, J. Arango-Lasprilla and H. Rogers, Necesidades familiares y su relación con las características psicosociales que presentan los cuidadores de personas con demencia, Psicología Desde el Caribe 26 (2010), 1-35.

[34] K. S. Multhaup and D. A. Balota, Generation effects and source memory in healthy older adults and in adults with dementia of the Alzheimer type, Neuropsychology 11(3) (1997), 382-91.

[35] N. W. Mulligan, Generation and hypermnesia, J Exp Psychol Learn Mem Cogn 27(2) (2001), 436-50.

[36] A. O'Brien, N. Chiaravalloti, J. C. Arango-Lasprilla, J. Lengenfelder and J. DeLuca, An investigation of the differential effect of self-generation to improve learning and memory in multiple sclerosis and traumatic brain injury, Neuropsychol Rehabil 17(3) (2007), 273-92.

[37] F. Ostrosky-Solís, A. Ardila and M. Rosselli, NEUROPSI Evaluación Neuropsicológica Breve en Español. Publingenio, editor. México2001. 
[38] B. J. Pesta, R. E. Sanders and M. D. Murphy, A beautiful day in the neighborhood: what factors determine the generation effect for simple multiplication problems? Mem Cognit 27(1) (1999), 106-15.

[39] B. J. Pesta, R. E. Sanders and R. J. Nemec, Older adults' strategic superiority with mental multiplication: a generation effect assessment, Exp Aging Res 22(2) (1996), 155-69.

[40] Z. F. Peynircioglu and E. Mungan, Familiarity, relative distinctiveness, and the generation effect, Mem Cognit 21(3) (1993), 367-74.

[41] B. Schefft, M. Dulay and J. Fargo, The use of a self-generation memory encoding strategy to improve verbal memory and learning in patients with traumatic brain injury, Appl Neuropsychol 15(1) (2008), 61-8

[42] N. Slamecka and P. Graf, The generation effect: Delineation of a phenomenon, Journal of Experimental Psychology: Learn- ing, Memory, \& Cognition 4(6) (1978), 592-604.

[43] S. Soraci, M. Carlin, R. Chechile, J. Franks, T. Wills and T. Watanabe, Encoding variability and cuing in generative processing, Journal of Memory and Language 41 (1999), 54159.

[44] L. Souliez, F. Pasquier, F. Lebert, P. Leconte and H. Petit, Generation effect in short-term verbal and visuospatial memory: comparisons between dementia of Alzheimer type and dementia of frontal lobe type, Cortex 32(2) (1996), 347-56.

[45] L. Taconnat and M. Isingrini, Cognitive operations in the generation effect on a recall test: role of aging and divided attention, J Exp Psychol Learn Mem Cogn 30(4) (2004), 827-37.

[46] R. Westmacott and M. Moscovitch, The contribution of autobiographical significance to semantic memory, Mem Cognit 31(5) (2003), 761-74. 
Copyright of NeuroRehabilitation is the property of IOS Press and its content may not be copied or emailed to multiple sites or posted to a listserv without the copyright holder's express written permission. However, users may print, download, or email articles for individual use. 